\title{
Anatomy of the fundus of the internal acoustic meatus - micro-computed tomography study
}

\author{
M. Kozerska, J. Skrzat \\ Department of Anatomy, Jagiellonian University, Collegium Medicum, Krakow, Poland
}

[Received 11 September 2014; Accepted 23 November 2014]

The aim of this paper was to present micro-computed tomography (micro-CT) high resolution images of the fundus of internal acoustic meatus (FIAM) and characterise the normal appearance of its singular areas which are places of passage of numerous anatomical structures. By using micro-CT we obtain detailed volume rendering images presenting topography of the FIAM in 3-dimensional (3D) space. We figured out that $3 D$ reconstructions obtained from micro-CT scans can precisely demonstrate all areas of the FIAM (facial nerve area, cochlear area, superior and inferior vestibular areas, singular foramen). Application of this technique allows finding out new anatomical structures like the foramen of the transverse crest, which is not described in literature. Hence, we estimated the size of each area of the FIAM by measuring their minimal and maximal diameter. In the studied material we did not find out any statistically significant difference between mean diameters calculated for infant and adult individuals. (Folia Morphol 2015; 74, 3: 352-358)

Key words: internal acoustic meatus, petrous bone, micro-computed tomography

\section{INTRODUCTION}

Internal acoustic meatus (IAM) is a canal which is terminated with a fundus located inside the pyramid of the temporal bone. The entire canal has a length of about $1 \mathrm{~cm}$ and extends laterally inside the bone. Lateral end of the IAM is formed by the thin cribriform plate of bone. This plate separates the cochlea and vestibule from the IAM, and is defined as a fundus of the internal acoustic meatus (FIAM). The FIAM constitutes also the medial wall of the labyrinth. The height and width of the FIAM range from 2.5 to $4.0 \mathrm{~mm}$ and from 2.0 to $3.0 \mathrm{~mm}$, respectively [5].

The FIAM transmits from the cranial cavity to ear the following structures: facial nerve, intermediate nerve, labyrinthine artery and vestibulocochlear nerve which divides near the lateral end of IAM into a two parts: a cochlear nerve and vestibular nerve. Within the FIAM runs horizontally transverse crest which separates the fundus into two parts: superior and inferior, as it is presented in Figure 1.

The superior part of the FIAM contains: facial nerve area (situated anteriorly) and superior vestibular area (situated posteriorly), whereas the inferior part contains: cochlear area (situated anteriorly), inferior vestibular area (situated posteriorly) and singular foramen (situated postero-inferiorly). Through the facial nerve area runs facial nerve and the intermediate nerve. The facial nerve area is separated from superior vestibular area by vertical osseous ridge termed as Bill's bar which forms the vertical crest. However, this structure is not always mentioned in the papers describing morphology of the FIAM, and therefore was not included in the schematic drawing presented in Figure 1.

Address for correspondence: M. Kozerska, MSc, Department of Anatomy, Collegium Medicum, Jagiellonian University, ul. Kopernika 12, 31-034 Kraków, Poland, e-mail: magdalena.kozerska@interia.pl 


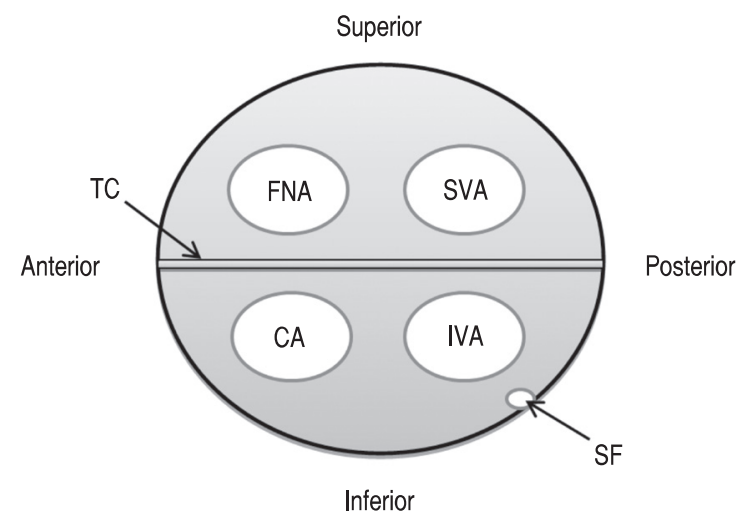

Figure 1. Schematic arrangement of the particular areas within the fundus of internal acoustic meatus; FNA — facial nerve area; SVA — superior vestibular area; CA — cochlear area; IVA — inferior vestibular area; SF — singular foramen; TC — transverse crest.

Superior vestibular area is a place of transition of the utriculoampullary nerve which originates from junction of the utricular nerve, anterior and lateral ampullar nerve.

Cochlear area, situated anteriorly in the inferior part of the FIAM is a place of passage of the cochlear nerve fibres which go through the fundus of the IAM from the modiolus of the cochlea. The cochlear nerve passes through the IAM along with the facial nerve and the vestibular nerve. The vestibular nerve originates from the superior and inferior vestibular nerves (passing through the corresponding fields within FIAM). In turn, the inferior vestibular area is a place for passing the saccular nerve.

The smallest area within the FIAM is occupied by the singular foramen which is located postero-inferiorly towards the inferior vestibular area, and transmits the posterior ampullar nerve. In spite of its small size this foramen is used as a landmark in certain surgical procedures $[1,11]$.

Up till now, in the anatomical and clinical studies IAM was investigated but details of the fundus morphology were not the primary scope of interest. Hence, this area has not been presented in a spatial manner with the aid of micro-computed tomography (micro-CT) or other imaging modality; thereby we undertook a study aimed on creating detailed computer reconstructions of the FIAM.

\section{MATERIALS AND METHODS}

Morphological study of anatomy of the FIAM was performed on 10 dry temporal bones: 5 samples derived from adult individuals of female sex, 2 samples were of male sex and 3 samples derived from infant skulls of unknown sex. All the examined bones were well preserved, presented normal anatomy and were not deformed.

The petrous part of the temporal bone was dissected and scanned with the micro-CT scanner (Skyscan 1172, N.V., Aartselaar, Belgium). The scanner was equipped with the $X$-ray detector: 11 Megapixel (4024 $\times 2680$ in total; $4000 \times 2400$ effective), 12 -bit digital $X$-ray camera with $24 \times 36 \mathrm{~mm}$ field of view. The $X$-ray source voltage was set to $80 \mathrm{kV}$ and current to $100 \mu \mathrm{A}$. The projection images were acquired over an angular range of $180^{\circ}$ with an angular step of $0.5^{\circ}$. In the resultant images pixel size was $27 \mu \mathrm{m}$. Projections were captured along the long axis of the petrous bone and reconstructed using a software NRECON ver. 1.6.5 SkyScan based on the Feldkamp algorithm.

Average number of the scans used to recreate volumetric reconstruction varied from 970 to 2093, depending on the size of the petrous part of the temporal bone. From the series of micro-CT scans we performed visualisation of the surface anatomy of FIAM. For this purpose we used volume rendering technique to present a 2 dimensional (2D) projection of a 3D discretely sampled data set produced by the micro-CT scanner and visualised in CTvox application. The CTvox application displays a set of reconstructed slices as a realistic 3D object with intuitive navigation and manipulation of both: object and camera and uses a clipping tool to produce cut-away views. The CTvox application is dedicated to realistic 3D visualisation of scanned objects by the SkyScan scanners and delivered by the Bruker Corporation (http://www. skyscan.be/products/downloads.htm).

To obtain clear and detailed images of the FIAM we adjusted experimentally transfer function which mapped opacity and grey values attributed to the voxels in the final reconstruction. By modifying the opacity we could control the visibility of the corresponding voxels and set how much they obscure more distant voxels.

Morphology of the FIAM was evaluated on the volumetric reconstructions of the petrous bone using clipping planes which position was interactively changed. Thus, we obtained convenient virtual section through the petrous bone presenting subsequent areas of the FIAM.

To estimate size of the each quadrant of the FIAM their minimum and maximum diameters were measured. For this purpose surface rendered 3D models 
Table 1. Mean values (in millimetres) of the diameters measured on surface-rendered 3-dimensional models of the fundus of internal acoustic meatus

\begin{tabular}{|c|c|c|c|c|c|c|c|c|c|c|}
\hline & \multicolumn{2}{|c|}{ SVA } & \multicolumn{2}{|c|}{ FNA } & \multicolumn{2}{|c|}{ CA } & \multicolumn{2}{|c|}{ IVA } & \multicolumn{2}{|c|}{ SF } \\
\hline & Min & Max & Min & Max & Min & Max & Min & Max & Min & Max \\
\hline Infant samples & 1.41 & 1.81 & 0.90 & 1.06 & 2.14 & 2.66 & 1.67 & 2.23 & 0.69 & 0.82 \\
\hline Adult samples & 1.20 & 1.43 & 1.05 & 1.46 & 2.32 & 2.75 & 1.34 & 1.70 & 0.66 & 0.95 \\
\hline
\end{tabular}

SVA — superior vestibular area; FNA — facial nerve area; CA — cochlear area; IVA — inferior vestibular area; SF — singular foramen

of the FIAM of each petrous bone were created from micro-CT scans dataset processed in CTAnalyser software. These models made visible a real aspects of the 3D structure of an object and were subjected as the OBJ file format into the Autodesk Meshmixer a free 3D modelling software equipped with tools for geometric measurements (available from http://meshmixer.com). Further, mean values of the diameters were estimated for facial nerve area (FNA), superior vestibular area (SVA), cochlear area (CA), inferior vestibular area (IVA) and singular foramen (SF) (Table 1). Diameters were measured with accuracy $\pm 100 \mu \mathrm{m}$ (Fig. 2).

Because of limited number of the studied temporal bones, we did not performed detailed analysis aimed on finding similarities or dissimilarities in morphological appearance of the FIAM between infant, male and female specimens. Thus, we compared only FIAM of infants (3 samples) versus adult male and female samples joined into one group (7 samples).

The Mann-Whitney test was computed to verify the null hypothesis which states that the diameters of the areas of the FIAM are equal.

\section{RESULTS}

The fundus of IAM was identified as lateral end of the IAM communicating the posterior cranial fossa with the labyrinth. The internal acoustic aperture being the inlet to IAM was easily recognized on the posterior surface of the pyramid of the temporal bone and reconstructed from micro-CT scans in all examined samples. These topographical relationships were visualised by volume rendering and overall morphology of the petrous bone with visible inlet to the IAM was presented in Figure 3.

The fundus of the IAM was observed as a discoid area which was divided by intersecting two bony ridges - the transverse crest and vertical crest. These structures delineated quadrants of unequal

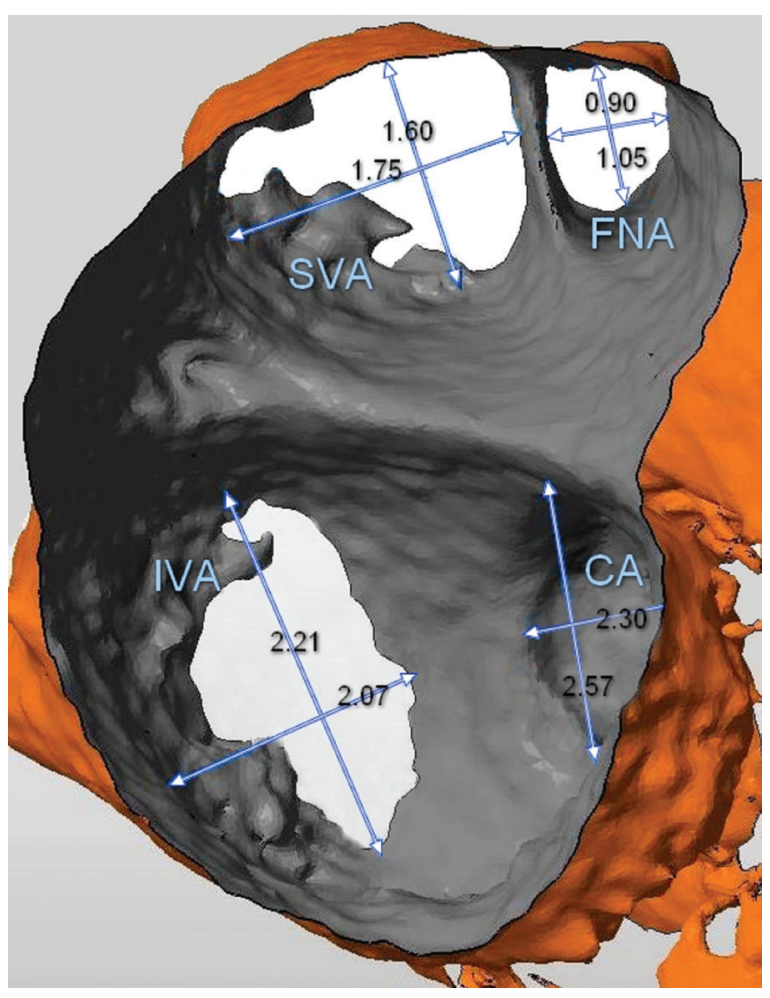

Figure 2. An example of measurements performed for each area of the fundus of internal acoustic meatus; SVA — superior vestibular area; IVA — inferior vestibular area; FNA — facial nerve area; $\mathrm{CA}$ - cochlear area. In this projection the area of the singular foramen is not visible.

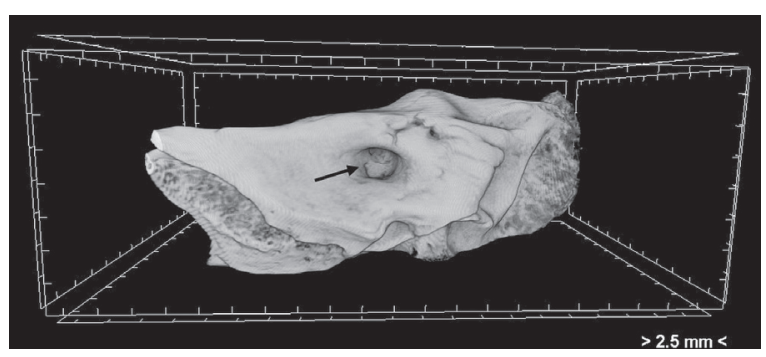

Figure 3. The anteromedial surface of the pyramid of the right temporal bone presented in a volume rendering image. Inlet to internal acoustic meatus is pointed by an arrow; in the depth the fundus of internal acoustic meatus is visible. 
size termed as FNA (anterosuperior quadrant), the CA (anteroinferior quadrant), SVA (posterosuperior quadrant) and IVA (posteroinferior quadrant). All these areas were imaged in volumetric reconstructions viewed at different angles. Their mutual orientation prevent from presenting entire morphology in a singular projection. Helical arrangement of the tractus spiralis foraminosus forces viewing the cochlear area at different angle than areas located on the posterior aspect of the FIAM. In turn, variable position of the singular foramen may be apparently caused by alteration of the viewing angle when it is observed simultaneously with the FIAM.

Normal anatomy of FIAM was demonstrated as a volume rendering image and presented in Figure 4. All described earlier fields within FIAM were observed in the examined samples and their position was consistent with generally accepted pattern (see diagram in Fig. 1). However, in the studied samples we observed following derogations in anatomy of individual fields within the FIAM:

- SVA and IVA were not a single aperture but in most cases consisted of few small apertures (Fig. 4);

- Different location of the singular foramen and distinct morphological appearance - well defined margin of the foramen or rounded and widened forming a recess (Fig. 5A, B).

In volume rendering images obtained from micro-CT we observed both septa of the FIAM: vertical (Bill's bar) and horizontal (transverse crest). The Bill's bar was appeared as osseous ridge of varying size (Fig. 6A, B). This structure, however not always described in scientific publications, was found in all examined samples.

In turn, the transverse crest did not reveal straight-line course (like it is usually presented in schematic drawings), but it rather had parabolic course. This was observable both in adult and infantile temporal bones.

Our attention was drawn by the transverse crests which derived only from the infant skulls. In all those cases we noticed a small foramen located posteriorly within the transverse crest. In this place the foramen was circular or elliptic (Fig. 6A, B). In adult temporal bones such foramen was not found within the transverse crest.

We found out that the minute foramen located in the transverse crest is the entrance to the osseous canal which runs to the wall of the vestibule. Diameter of the canal gradually decreases towards the vestibule. In one case this canal was divided into

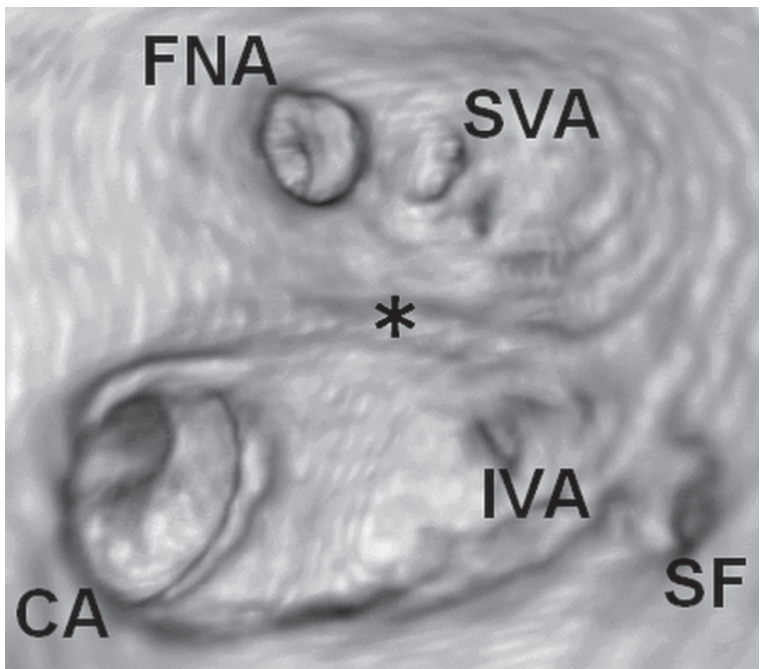

Figure 4. Volume rendering image of the fundus of internal acoustic meatus located in right temporal bone of an adult individual of female sex; FNA — facial nerve area; SVA — superior vestibular area; CA — cochlear area; IVA — inferior vestibular area; SF — singular foramen. The transverse crest is marked by the asterisk.
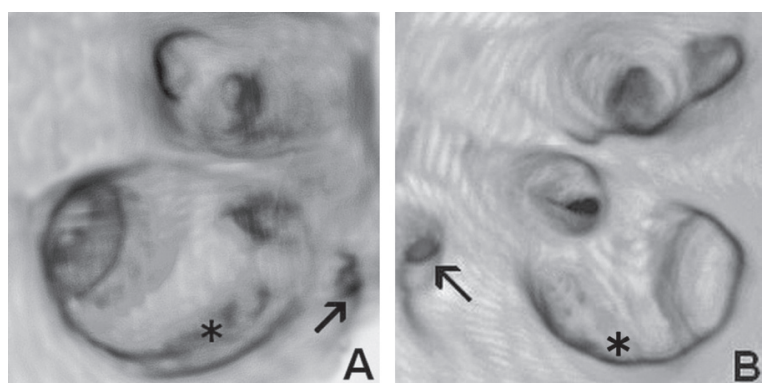

Figure 5. Examples of distinct microarchitecture of the fundus of internal acoustic meatus in adults; A. Right female temporal bone; B. Left male temporal bone. The most important differences concern the shape of the tractus spiralis foraminosus (marked by the asterisk) and position of the singular foramen (pointed by the arrow).
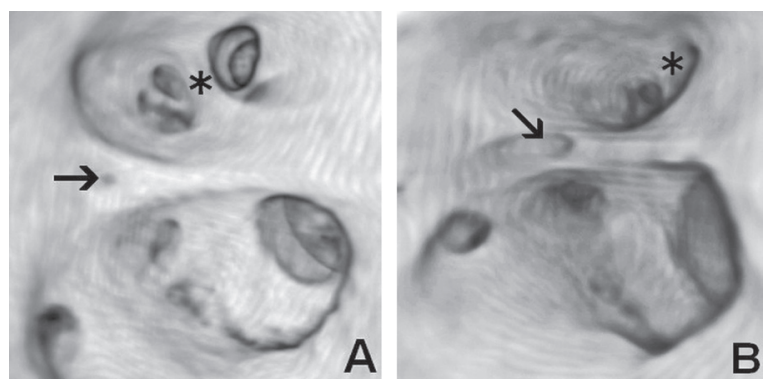

Figure 6. Anatomy of the fundus of internal acoustic meatus of 2-year-old child (A) and of 6-year-old child (B) characterizes vascular foramen of the transverse crest (indicated by the arrow). The vertical crest (Bill's bar) is marked by the asterisk.

two separate canaliculi which run to the superior vestibular canal, instead of direct connection with the vestibule. We also spotted a case in which the 

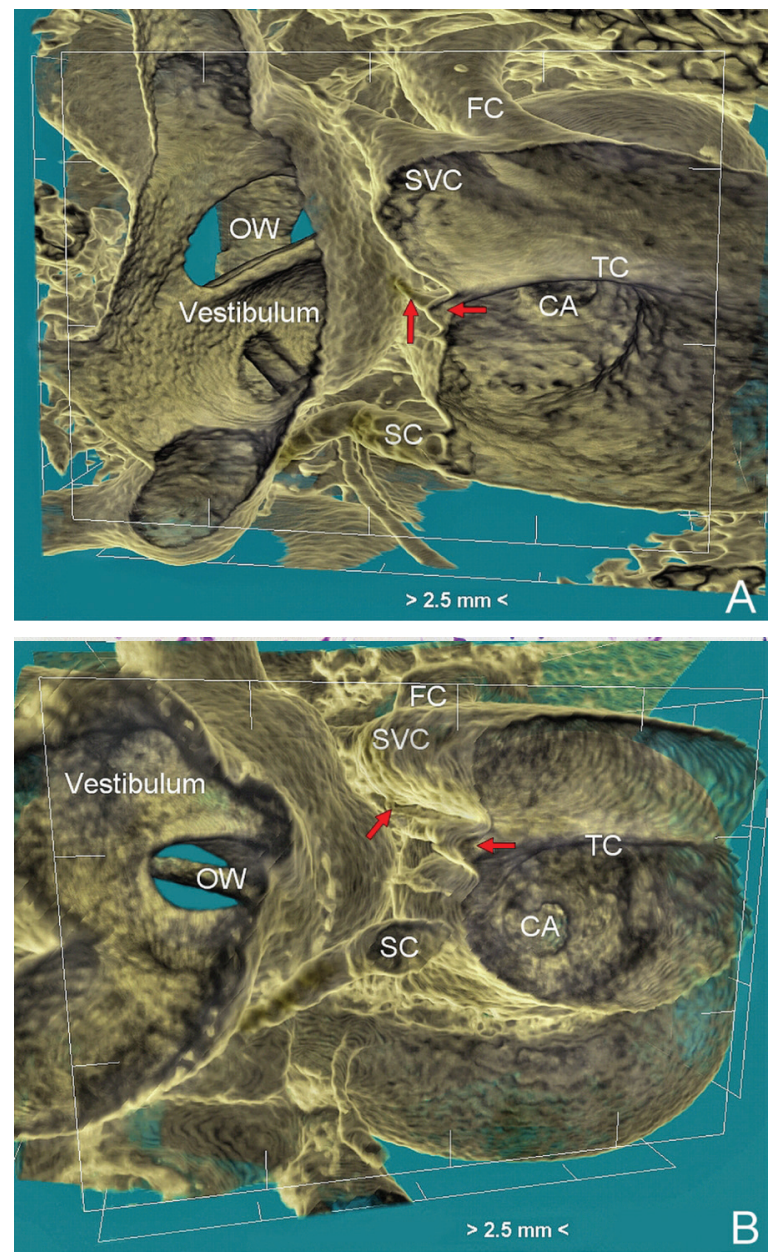

Figure 7. Three-dimensional representation of the fundus of internal acoustic meatus and related neurovascular canals; A. Canaliculus of the transvers crest (TC) (indicated by arrows) connected the vestibule and the internal acoustic meatus; B. Canaliculi (indicated by arrows) running from the foramen located within the TC to the superior vestibular canal (SVC); $\mathrm{OW}$ - oval window; $F C$ - facial canal; SC — singular canal; CA — cochlear area.

canal was obliterated, and its ending was of conical shape. Observed morphological variety of the canaliculi of the transverse crest is demonstrated in Figures 6 and 7 .

The Mann-Whitney test did not confirm that differences between mean values of the diameters of the selected areas of the FIAM in infant and adult samples are statistically significant. Therefore, we presume that observed discrepancies between calculated parameters are rather related to biological variation than are effect of age influence in the studied material. Due to limited and uneven number of specimens, these results cannot be extended on general population and should be verified on large clinical series.

\section{DISCUSSION}

In contemporary literature there is a lack of images presenting FIAM morphology in high resolution. Up till now, most of the studies showed morphology of the FIAM using operating microscope or this region of the temporal bone was visualised by clinical CT scanners. Thereby, previous results were limited rather to analysis of the appearance of the entire IAM and not exactly its fundus. For instance, Marques et al. [10] has examined the shape of the IAM and established that it could be funnel-shaped (the most common in children and adults), cylindrical or bud-shaped which is the least represented.

The other aspect of the anatomical studies of this region of the temporal bone relates to the bony neural canals (for the CA, FNA, SVA, and IVA) which arise from the FIAM. In 1999 Fatterpekar et al. [4] presented the topography and morphology of these canals in axial and coronal 1-mm-thick CT scans and performed measurements. Since that time, there were no studies focusing on morphological analysis of the individual fields within the FIAM. Only cochlear area and the cochlear nerve canal were the subject of intensive morphometric analysis, because it is well visible on the CT scans and can be easily measured. Moreover, the cochlear nerve canal has tremendous clinical significance, because changes of its diameter may be the reason of cochlear nerve deficiency which is believed to be one of the causes of sensorineural hearing loss $[8,14]$.

Clinical CT provides images where small canals of the temporal bone can be visualised. Nevertheless, their appearance is not always clear and sometimes may be confused with fractures. Therefore evaluation of their morphology based on CT scans seems to be problematic and biased with error, if any measurements are performed on such images. To our knowledge, the only paper that presents measurements of the individual fields within the FIAM was published in 1999 [4]. Perhaps it was dictated by limitations in presenting osseous details, which dimensions range in millimetre scale or even less. Moreover, due to their intricate shape and various spatial orientations, 3D imaging techniques should be used for their visualisation. These obstacles can be overcome by usage of micro-CT which may deliver CT scans of extremely high resolution, which provides an accurate imaging of anatomical details.

In current study application of micro-CT allowed visualisation of foramen located within the margin of the transverse crest and traced the ca- 
nal emerging from it. Because of small diameter $(<0.50 \mathrm{~mm})$, this foramen cannot be noticed in clinical CT scans or could be hardly visible. So far, we have not found any information on the presence and importance of the foramen of the transverse crest and related osseous canals. We presume that such foramen and canal transmit blood vessels vascularising the wall of the vestibule or the superior vestibular canal. It could be a branch of the labyrinthine artery that runs between facial and cochlear nerves, emerging at the fundus of the IAM, and splits into three terminal branches. One of these branches, namely the anterior vestibular artery, could penetrate the FIAM within foramen of the transverse crest, subsequently ending in the vestibule by division into smaller arterioles $[2,15]$.

Another possible content of the foramen of the transverse crest could be related to anastomoses between the facial, vestibular and cochlear nerves that may occur before entering or after exiting the FIAM. Existence of vestibulocochlear and vestibulofacial connections within the IAM has been described in literature $[12,13]$.

Current and future researches should be aimed not only at presenting morphology of the FIAM in high resolution but also at performing accurate measurements using computing methods based on imaging techniques and software modelling. Knowledge on the detailed anatomy and topography of the singular quadrants of the FIAM and bony canals emerging from them is necessary during evaluation the temporal bone trauma and congenital anomalies affecting the individual nerves as well as during neurootologic surgical procedures $[6,9]$. For instance, the singular foramen is used as a landmark for certain surgical procedures on the internal acoustic meatus and labyrinth, such as retrosigmoid acoustic neuroma surgery and transcochlear cochleovestibular neurectomy [11]. Sometimes tumour removal at the fundus must be done blindly because field of view of the entire fundus is restricted, thus the vestibulocochlear, facial nerve and labyrinthine artery can be exposed at additional risk of damage $[3,7]$. Therefore, it is important to implement and develop new algorithms dedicated for accurate and spatial visualisation of the FIAM and combine $3 \mathrm{D}$ reconstructions generated from a stack of micro-CT scans with radiological images obtained from clinical researches. Advanced methods of spatial imaging can help to understand inner ear anatomy and enhance planning and performing surgical operations.

\section{CONCLUSIONS}

From our study we conclude that topography of the singular areas within the FIAM presented in the schematic drawings is strongly simplified. Clinical tomography is not able to reflect mutual relationship between osseous structures of the FIAM. Micro-CT is an adequate technique for imaging surface topography of the FIAM and evaluation of its architecture. By providing high-quality $2 \mathrm{D}$ and $3 \mathrm{D}$ reconstructions, new anatomical structures can be captured within the FIAM. Hence, micro-CT scans dataset can be used to build accurate surface rendered 3D models of the FIAM and perform on them geometrical measurements.

\section{ACKNOWLEDGEMENTS}

The research was carried out with the equipment purchased thanks to the financial support of the European Regional Development Fund in the framework of the Polish Innovation Economy Operational Program (contract no. POIG.02.01.00-12-023/08).

The authors would like to thank to Bartosz Leszczyński from the Department of Medical Physics, M. Smoluchowski Institute of Physics, Jagiellonian University for performing microcomputed tomography of the petrous bones.

The study was conducted with approval (KBET/109/B/2012) of the Bioethics Committee of the Jagiellonian University. We declare that we have no competing interests.

\section{REFERENCES}

1. Agirdir BV, Sindel M, Arslan G, Yildirim FB, Balkan El, Dinç O (2001) The canal of the posterior ampullar nerve: an important anatomic landmark in the posterior fossa transmeatal approach. Surg Radiol Anat 23 : 331-334.

2. Brunsteins DB, Ferreri AJ (1995) Microsurgical anatomy of arteries related to the internal acoustic meatus. Acta Anat (Basel), 152: 143-150.

3. Driscoll CLW, Jackler RK, Pitts LH, Banthia V (2000) Is the entire fundus of the internal auditory canal visible during the middle fossa approach for acoustic neuroma? Am J Otol, 21: 382-388.

4. Fatterpekar GM, Mukherji SK, Lin Y, Alley JG, Stone JA, Castillo M (1999) Normal canals at the fundus of the internal auditory canal: CT evaluation. J Comput Assist Tomogr, 23: 776-780.

5. Farahani RM, Nooranipour M, Nikakhtar KV (2007) Anthropometry of internal acoustic meatus. Int J Morphol, 25: 861-865. 
6. Gonzalez LF, Lekovic GP, Porter RW, Syms MJ, Daspit CP, Spetzler RF (2004) Surgical approaches for resection of acoustic neuromas. Barrow Quarterly, 20: 4.

7. Haberkamp TJ, Meyer GA, Fox M (1998) Surgical exposure of the fundus of the internal auditory canal: anatomic limits of the middle fossa versus the retrosigmoid transcanal approach. Laryngoscope, 108: 1190-1194.

8. Li Y, Yang J, Liu J, Wu H (2014) Restudy of malformations of the internal auditory meatus, cochlear nerve canal and cochlear nerve. Eur Arch Otorhinolaryngol, DOI: 10.1007/ s00405-014-2951-4 (in press).

9. Marchioni D, Alicandri-Ciufelli M, Mattioli F, Nogeira JF, Tarabichi M, Villari D, Presutti L (2012) From external to internal auditory canal: surgical anatomy by an exclusive endoscopic approach. Eur Arch Otorhinolaryngol, 270: 1267-1275.

10. Marques SR, Ajzen S, D'Ippolito G, Alonso L, Isotani S, Lederman H (2012) Morphometric analysis of the internal auditory canal by computed tomography imaging. Iran J Radiol, 9: 71-78.

11. Muren C, Wadin K, Dimopoulos P (1991) Radioanatomy of the singular nerve canal. Eur Radiol, 1: 65-69.

12. Ozdoğmuş O, Sezen O, Kubilay U, Saka E, Duman U, San T, Cavdar S (2004) Connections between the facial, vestibular and cochlear nerve bundles within the internal auditory canal. J Anat, 205: 65-75.

13. Tian GY, Xu DC, Huang DL, Liao H, Huang MX (2008) The topographical relationships and anastomosis of the nerves in the human internal auditory canal. Surg Radiol Anat, 30: 243-247.

14. Yan F, Li J, Xian J, Wang Z, Mo L (2013) The cochlear nerve canal and internal auditory canal in children with normal cochlea but cochlear nerve deficiency. Acta Radiol, 54: 292-298.

15. Zhang K, Wang F, Zhang Y, Li M, Shi X (2002) Anatomic investigation of the labyrinthine artery. Zhonghua $\mathrm{Er} \mathrm{Bi}$ Yan Hou Ke Za Zhi, 37: 103-105. 